\title{
Coupling Interaction and Physiological Metrics for Interaction Adaptation
}

\author{
Luís Duarte and Luís Carriço \\ LaSIGE \& Department of Informatics, University of Lisbon \\ Edifício C6, Campo-Grande, 1749-016 Lisboa \\ lduarte@lasige.di.fc.ul.pt, lmc@di.fc.ul.pt
}

\begin{abstract}
We present an adaptation system whose goal is to provide users with interaction experiences tailored to their current physiological status and performance. The system captures emotion, motion and application related metrics to proactively adjust the available interaction patterns. Interacting in different environments - stationary/mobile - or under different emotional status - relaxed/stressed - can affect performance, engagement and enjoyment. This contribution describes the initial design steps in the creation of an interaction adaptation engine.
\end{abstract}

Keywords: Physiological Signals, Adaptation, User Performance.

\section{Motivation}

Physiological user interfaces are typically employed to perceive real body signals from users, while utilizing that information during the interaction period [7]. These interfaces are mainly used to determine motion [2] (through muscle tension detection, video capture or accelerometers) or emotion [6] (through skin conductivity or heart rate variance). Human beings are capable of expressing both observable (e.g. blushing, frowning, etc.) and concealed (e.g. increasing heart beat rate, altering body temperature, etc.) reactions to certain events. While analysts and the most recurrently used mechanisms are able to retrieve a significant amount of data with traditional assessment techniques (e.g. camera recording, ethnography, questionnaires, etc.), these are considered highly subjective and possess an interesting negative effect for the former [3]: they typically fail to address complex interaction patterns. On the other hand, Rowe [5] states that some of the advantages of these interfaces are linked with being multi-dimensional, proving to be capable of providing alternative views on different issues. Lastly, physiological signals are typically continuously gathered, enabling a faster and more accurate detection of emotional or workload shifts.

However, one domain in which physiological interaction mechanisms have failed to prosper in is mobile environments. There are multiple examples of studies on the use of such interaction mechanisms in desktop or stationary settings in a diversity of areas, ranging from entertainment to healthcare. The most recent examples of the use of physiological mechanisms in mobile settings stem from the NikeRunning [4] program and from a system which collects physiological data from users with the aim of improving positive emotions in their daily lives [8]. Nevertheless, neither of these 
approaches addresses some of the most important challenges of mobile environments: constant transitions to/from different settings, interaction with peers, the device's characteristics, to cite a few.

Taking these factors into account and based on a set of experiments we have been performing to assess user behavior and interaction patterns using physiological indicators [1], we envisioned an adaptation engine for both stationary and mobile settings, which combines application log data with physiological measures to better tailor interaction styles and user interfaces to the operators' current status.

\section{Adaptation Engine}

The adaptation engine encompasses the use of physiological assessment mechanisms to evaluate user performance. Our approach comprises the following components:

- Emotional Activity Module: is related with shifts in determined biological signals, such as heart beat rate or skin conductance. Since these are two of the most researched metrics, the purpose is to identify periods of increased stress or anxiety. With this module we intend on providing the necessary mechanisms to pro-actively improve user experience and provide users with a balanced interaction.

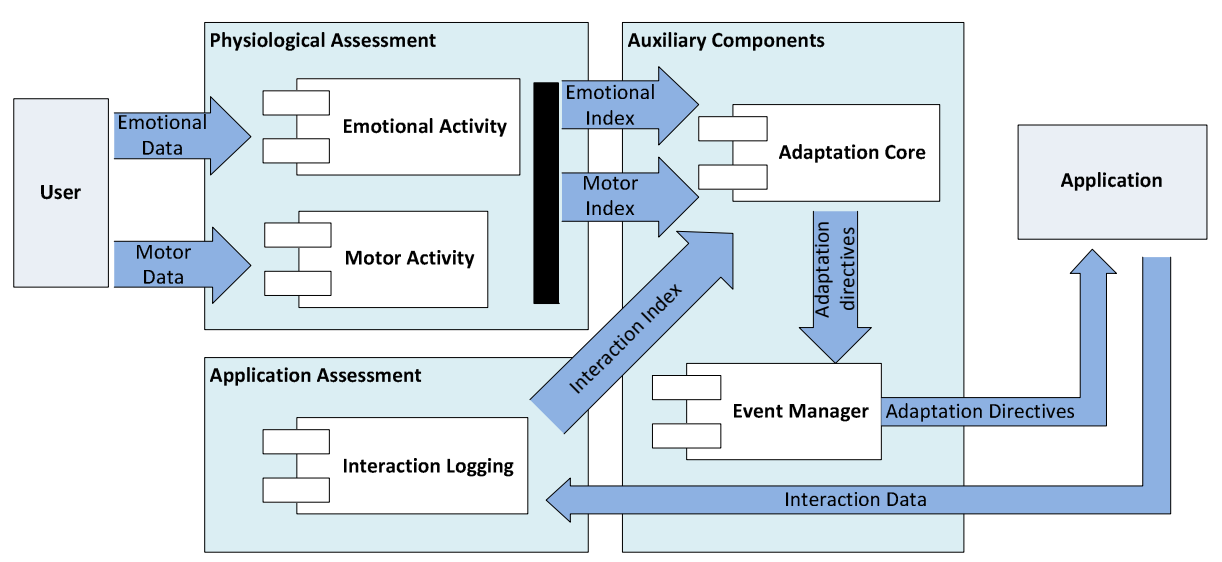

Fig. 1. Adaptation Engine's architecture

- Motor Activity Module: is concerned with the identification of user movement. In particular, it identifies whether a user is walking, if he/she waved his/her arms, etc. The coupling of such module with the emotional one allows us to partially differentiate motor activity which is related with interaction patterns from that which results from user locomotion or other external variables.

- Interaction Logging: collects relevant data of user interaction with the application. The module encompasses metrics which may range from 
number of clicks on a specified interval, idle time to number of hops to reach a certain feature. Coupling these metrics with physiological data is paramount to identify emotional or motor fluctuations which are a result of interacting with applications or devices, from those which stem from external interventions.

- Adaptation Core: composed by data structures which store gathered data and a set of indexes and weights for each source, indicating how influential the data source is to the application or a control.

- Event Manager: provides a set of services which ensure the separation between all engine logic from the user interface, by using the Windows Message Queue as a channel.

\section{Envisioned Scenario}

The following example is a mere possibility of applying the engine in domains with widespread popularity. Entertainment, and in particular videogames, is a well suited thematic for adaptation examples.

\subsection{Motion Game}

Let us imagine a game in which players need to perform a series of gestures in order to accomplish certain tasks - a common setting with the increased popularity of motion-based interaction controls from major manufacturers. In this game, John, needs to perform the gesture in a fixed time window (see Figure 2) to cast a spell and defeat an enemy.
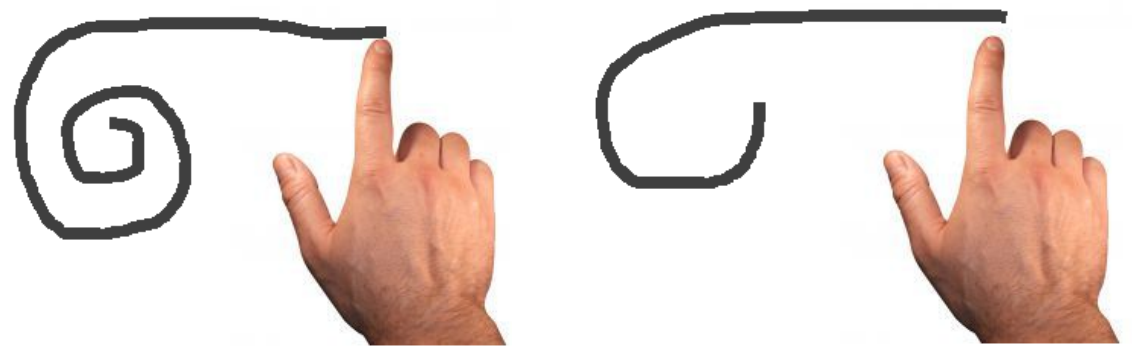

Fig. 2. Adaptation Engine's architecture

However, John is feeling tired from work and is currently struggling to achieve the desired motion in the appropriate time frame. While his accuracy is being recorded, the adaptation engine was capable of checking that his heartbeat rate has significantly increased since he started attempting to perform the gesture. Given the overall setting of a high biological signal being verified and diminished user accuracy, the game adapts itself to allow for simple gestures to be inputted. 
This is a simple example of an adaptation process which encompasses user physiological data and interaction logging to change the way an individual interacts. Other possible scenarios may include difficulty adaptation, automatic scaling of interaction controls, interactive alternatives to the primary input methods, etc.

\section{Future Work}

It is our belief that as, a few years ago, accelerometers and other sensors were integrated into our everyday lives' devices, so will physiological interaction mechanisms in the future. The contribution from this ongoing work is far from accurately identifying emotions or exploring how to integrate physiological sensors into cell phones, but rather exploring scenarios and understanding user behavior and interaction patterns so that application design is tailored to our needs.

Future work encompasses finalizing the implementation of the adaptation engine and carrying our various experiments to assess how users interact in order to provide more realistic and tailored adaptation procedures in the engine.

Acknowledgments. This work was funded by FCT, through Individual Scholarship SFRH / BD / 39496 / 2007, through project PTDC/EIA-EIA/103676/2008 (InSIThe) and the Multiannual Funding Programme.

\section{References}

1. Duarte, L., et al.: Physiological Data Gathering in Mobile Environments. In: Procs. of UBICOMP 2010, Copenhagen, Denmark (2010)

2. Kwon, D., et al.: Combining body sensors and visual sensors for motion training. In: Procs. of ACE 2005, pp. 94-101. ACM, New York (2005)

3. Meehan, M., et al.: Physiological measures of presence in stressful virtual environments. In: Procs. of SIGGRAPH 2002, pp. 645-652. ACM, New York (2002)

4. NikeRunning, http://nikerunning.nike.com

5. Rowe, D., et al.: Heart rate variability: indicator of user state as an aid for human-computer interaction. In: Procs. of CHI 1998, pp. 480-487. ACM, New York (1998)

6. Shi, Y., et al.: Galvanic skin response (GSR) as an index of cognitive load. In: CHI EA 2007, pp. 2651-2656. ACM, New York (2007)

7. Tomida, T., et al:: The communication entertainment content by using entrainment phenomenon and bio-feedback. In: Procs. of ACE 2007, pp. 286-287. ACM, New York (2007)

8. Vaara, E., et al.: Mirroring bodily experiences over time. In: CHI EA 2009, pp. 4471-4476. ACM, New York (2009) 